\title{
Longus Colli Tendinitis in a Patient Presenting with Neck Pain and Acute Systemic Inflammation
}

\author{
Takayasu Uchida, Mami Kanzaki, Toshiyuki Kakumoto and Yoshikazu Uesaka
}

\begin{abstract}
:
The diagnosis of longus colli tendinitis (LCT) is sometimes challenging, especially when laboratory data show marked inflammation and neuroimaging studies do not indicate calcification within the tendon of the longus colli muscles. We herein report a case of LCT that presented with elevated inflammation parameters without calcification on imaging. Findings characteristic of LCT, such as prevertebral hyperintensity areas on T2-weighted images and no signs of purulent diseases, informed our diagnosis of LCT. Enhanced computed tomography and magnetic resonance imaging are useful procedures when diagnosing LCT after ruling out other critical purulent diseases.
\end{abstract}

Key words: longus colli tendinitis (LCT), prevertebral effusion, retropharyngeal abscess, calcium hydroxyapatite

(Intern Med 57: 2759-2761, 2018)

(DOI: 10.2169/internalmedicine.0160-17)

\section{Introduction}

Longus colli tendinitis (LCT) is a calcium hydroxyapatite deposition disease first reported by Hartley in 1964 (1). The clinical findings of LCT include neck pain, a limited range of motion of the neck, neck stiffness, odynophagia, dysphagia, sore throat, and a fever (2). Conservative treatments, including anti-inflammatory drugs and immobilization of the neck, are recommended. It is sometimes difficult to diagnose LCT, as these presentations are also seen in patients with retropharyngeal infection or spondylitis. Enhanced computed tomography (CT) and magnetic resonance imaging (MRI) are useful for differentiating these lifethreatening diseases from LCT. On neuroimaging studies, calcifications located along the longus colli, retropharyngeal effusion, no signs of abscess, and no bony changes are characteristic of LCT (3). In most cases, blood tests show mildly elevated inflammation values (4).

We herein report a case of LCT with marked inflammation and no calcification within the tendon of the longus colli muscles on imaging studies.

\section{Case Report}

A 24-year-old man presented with an 8-day history of a fever and severe progressive neck pain and a 3-day history of difficulty opening his mouth. His medical history was deemed noncontributory. On a physical examination, his vital signs were within normal limits, except for his elevated body temperature of $38.5^{\circ} \mathrm{C}$. The range of motion of his neck was significantly restricted in all directions due to pain. Bilateral conjunctival injection, cervical lymphadenopathy, and pharyngeal erythema without exudates or deviation of the uvula were also noted at presentation. Laboratory data revealed a white blood cell count of $11,000 / \mathrm{mm}^{3}$, a Creactive protein (CRP) value of $22.5 \mathrm{mg} / \mathrm{dL}$, and an erythrocyte sedimentation rate (ESR) of $110 \mathrm{~mm} / \mathrm{h}$. Liver enzymes, creatinine and thyroid function values were within normal limits. Blood culture results were negative in two samples taken at different sites. There were no remarkable serological findings that indicated infection by the Epstein-Barr (EB) virus, cytomegalovirus, herpes simplex virus, or the herpes zoster virus. Screening tests for other infectious diseases, such as hepatitis B and C, tuberculosis, syphilis, and HIV, were negative. In addition, the results of screening 


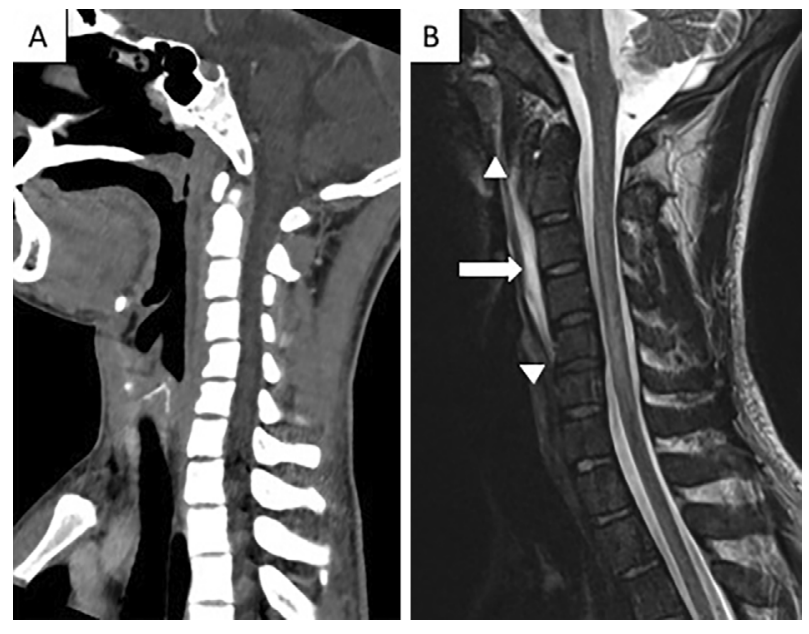

Figure. A: The enhanced CT scan of the neck on admission, which shows prevertebral effusion without calcification. B: A sagittal section of the T2-weighted cervical MRI scan, which shows prevertebral effusion at the $\mathrm{C2}-\mathrm{C} 5$ level (indicated with a white arrowhead).

tests for rheumatologic disorders, such as antinuclear antibodies, rheumatoid factors, anti-CCP antibodies, anti-dsDNA antibodies, anti-Sm antibodies, anti-Ro/SSA, anti-La/SSB, proteinase 3 anti-neutrophil cytoplasmic antibody (PR3ANCA), and myeloperoxidase (MPO)-ANCA, were also negative. The cerebrospinal fluid values were within the normal range.

Contrast-enhanced CT showed prevertebral soft tissue swellings with no signs of retropharyngeal abscess or calcification (Figure A). MRI demonstrated prevertebral hyperintense areas between $\mathrm{C} 2-\mathrm{C} 5$ on $\mathrm{T} 2$-weighted images and no signs of retropharyngeal infection or spondylitis on T1weighted images (Figure B). We asked an otolaryngologist to perform an examination with a fiberscope, which revealed no obvious evidence of pharyngeal abscess. Consequently, a diagnosis of LCT was made.

The patient received $180 \mathrm{mg}$ of loxoprofen and $200 \mathrm{mg}$ of tramadol hydrochloride daily for the pain. His symptoms improved dramatically immediately after he received the medications. He was also started on a 14-day course of IV ampicillin-sulbactam, followed by $500 \mathrm{mg}$ of oral levofloxacin daily for 5 days prior to admission. MRI performed eight days after the commencement of treatment showed that the prevertebral lesion seen at his initial presentation had almost completely disappeared. He was discharged after 14 days with almost full recovery from his symptoms.

\section{Discussion}

LCT, also known as retropharyngeal calcific tendinitis, is a benign disease caused by calcium hydroxyapatite deposition within the tendon of the longus colli muscles. Its clinical presentations include neck pain, a limited range of motion of the neck, neck stiffness, odynophagia, dysphagia, sore throat, and a fever. On imaging studies, such as CT and
MRI, calcification along the longus colli is often noted. A review of the literature that included 242 cases of acute calcific vertebral tendinitis reported by 104 articles revealed that the mean age was 43 years (21-81 years), the CRP values in 21 cases ranged from 0.3 to $91.3 \mathrm{mg} / \mathrm{dL}$ (mean 23.66 $\mathrm{mg} / \mathrm{dL}$ ), and the ESR in 45 cases ranged from 5 to $98 \mathrm{~mm} / \mathrm{h}$ (mean $31.57 \mathrm{~mm} / \mathrm{h}$ ) based on blood tests (5). The present case showed severe inflammation on blood tests and no calcification on imaging. The CRP value of $22.5 \mathrm{mg} / \mathrm{dL}$ and ESR of $110 \mathrm{~mm} / \mathrm{h}$ were particuarly high compared with previous cases. Differential diagnoses included meningitis, retropharyngeal abscess, pyogenic spondylitis, cervical disc herniation, fracture dislocation, neoplasm, and Crowned dens syndrome. Crowned dens syndrome is also a rare calcium pyrophosphate deposition disease, showing calcification of the cruciform ligament on imaging studies. However, the mean age at the diagnosis of Crowned dens syndrome was reported to be 60 years, which is older than that for LCT (6).

Some cases of LCT, such as our own, do not show calcification on imaging studies, although most do (5). In cases without calcification, prevertebral effusion on T2-weighted images is important for achieving a diagnosis of LCT. A previous study reported that the extent of calcification is not associated with the severity of clinical symptoms (4). When making a diagnosis of LCT in cases with marked inflammation and no calcification on imaging, it is especially important to rule out other purulent diseases, such as retropharyngeal abscess and pyogenic spondylitis, as these diseases often have lethal outcomes. Enhanced CT is recommended over MRI as a first-line imaging procedure, due to the lower medical costs and ability to rule out the possibility of purulent diseases, as discussed above (2). However, MRI is more sensitive than CT in detecting vertebral osteomyelitis and epidural abscess (7). Thus, imaging procedures should be chosen depending on the patient's clinical presentation, although performing both enhanced CT and MRI is the ideal method for ruling out serious diseases when diagnosing LCT.

Several limitations associated with the present study warrant mention. Enhanced CT did not reveal any obvious signs of retropharyngeal abscess. However, purulent diseases could not be ruled out completely because the patient had already been given antibiotics for five days prior to his admission. We continued administering antibiotics during the admission. This may have caused the false-negative results of the blood cultures and the enhanced CT scan of the neck. In addition, the cause of LCT is unknown, although some explanations, such as recent injury or ischemia, have been proposed (8). Our case had experienced neck trauma a few months prior to presentation, which may have triggered the development of this disease.

The authors state that they have no Conflict of Interest (COI).

Financial Support 
Yoshikazu Uesaka served as the primary investigator and received institutional support from the Okinaka Memorial Institute for Medical Research. He is funded by the Ministry of Health, Labour and Welfare of Japan as a member of its "Wake-up stroke" and "Subacute myelo-optico-neuropathy" research team.

\section{References}

1. Hartley J. Acute cervical pain associated with retropharyngeal calcium deposit: a case report. J Bone Joint Surg Am 46: 1753-1754, 1964.

2. Park R, Halpert DE, Baer A, Kunar D, Holt PA. Retropharyngeal calcific tendinitis: case report and review of the literature. Semin Arthritis Rheum 39: 504-509, 2010.

3. Paik NC, Lim CS, Jang HS. Tendinitis of longus colli: computed tomography, magnetic resonance imaging, and clinical spectra of 9 cases. J Comput Assist Tomogr 36: 755-761, 2012.
4. Offiah CE, Hall E. Acute calcific tendinitis of the longus colli muscle: spectrum of CT appearances and anatomical correlation. Br J Radiol 82: e117-e121, 2009.

5. Shawky A, Elnady B, El-Morshidy E, Gad W, Ezzati A. Longus colli tendinitis. A review of literature and case series. SICOT J 3: 48, 2017.

6. Godfrin-Valnet M, Godfrin G, Godard J, et al. Eighteen cases of crowned dens syndrome: Presentation and diagnosis. Neurochirurgie 59: 115-120, 2013.

7. An HS, Seldomridge JA. Spinal infections: diagnostic tests and imaging studies. Clin Orthop Relat Res 444: 27-33, 2006.

8. DeMaeseneer M, Vreugde S, Laureys S, Sartoris DJ, DeRidder F, Osteaux M. Calcific tendinitis of the longus colli muscle. Head Neck 19: 545-548, 1997.

The Internal Medicine is an Open Access article distributed under the Creative Commons Attribution-NonCommercial-NoDerivatives 4.0 International License. To view the details of this license, please visit (https://creativecommons.org/licenses/ by-nc-nd/4.0/).

(C) 2018 The Japanese Society of Internal Medicine Intern Med 57: 2759-2761, 2018 